\title{
HIGHER ORDER NONLOCAL NONLINEAR BOUNDARY VALUE PROBLEMS FOR FRACTIONAL DIFFERENTIAL EQUATIONS
}

\author{
Rahmat Ali Khan
}

\begin{abstract}
In this paper, we study the method of upper and lower solutions and develop the generalized quasilinearization technique for the existence and approximation of solutions to some three-point nonlocal boundary value problems associated with higher order fractional differential equations of the type

${ }^{c} \mathcal{D}_{0+}^{q} u(t)+f(t, u(t))=0, t \in(0,1)$

$u^{\prime}(0)=\gamma u^{\prime}(\eta), u^{\prime \prime}(0)=0, u^{\prime \prime \prime}(0)=0, \ldots, u^{(n-1)}(0)=0, u(1)=\delta u(\eta)$,

where, $n-1<q<n, n(\geq 3) \in \mathbb{N}, 0<\eta, \gamma, \delta<1$ and ${ }^{c} D_{0+}^{q}$ is the Caputo fractional derivative of order $q$. The nonlinear function $f$ is assumed to be continuous.
\end{abstract}

\section{Introduction}

The theory of boundary value problems (BVPs in short) for ordinary differential equations is well studied and numerous results can be found in the literature dealing with the solvability of boundary value problems. In contrast, the theory of BVPs corresponding to nonlinear fractional differential equations is in the initial stage of development and many aspects of the theory need to be investigated. For the recent development in the theory of existence of solutions to BVPs associated with fractional differential equations, we refer the readers to $[2,3,4,6,9,12,13,15,18,19,20,21,22,25]$ and the references therein. In the above cited references, the classical tools of functional analysis have been used to establish sufficient conditions for existence results. However, the theory of upper and lower solutions and comparison results corresponding to BVPs associated with fractional differential equations is in the initial stage and as far as I know, only few results can be found in the literature dealing with the upper and lower solutions method $[1,5,16,23,24]$. In these references, boundary value problems corresponding to fractional differential equations of order

Received August 26, 2011; Revised December 29, 2011.

2010 Mathematics Subject Classification. 26A33, 34K05, 34K07.

Key words and phrases. boundary value problems, fractional differential equations, threepoint boundary conditions, upper and lower solutions, generalized quasilinearization. 
$1 \leq q<2$ subject to two point boundary conditions are studied. Some of them study BVPs with impulses as well. Our problem is different from the previously studied problems in the sense that we study more general higher order BVPs corresponding to fractional differential equations of order $n-1 \leq q<n, n \geq 3$ subject to multi-point boundary conditions of the type $u^{\prime}(0)=\gamma u^{\prime}(\eta), u^{\prime \prime}(0)=$ $0, u^{\prime \prime \prime}(0)=0, \ldots, u^{(n-1)}(0)=0, u(1)=\delta u(\eta)$. The method of quasilinearization has already been developed for some initial value problems associated with fractional differential equations $[7,8,14,17]$ but results dealing with quasilinearization to boundary value problems for fractional differential equations can hardly be seen in the literature $[10,11]$. In [10], BVPs corresponding to fractional differential equation of order $1<q<2$ subject to boundary conditions $u^{\prime}(0)=0, u(1)=\xi u(\eta)$ are studied while in [11], the problem with $2<q<3$ subject to boundary conditions $u(0)=u^{\prime}(0)=0, u(1)=\sum_{i=1}^{n-2} \xi_{i} u\left(\eta_{i}\right)$ is studied. The purpose of this paper is to develop comparison results and upper and lower solutions method for the existence of solution to boundary value problem (BVP) for higher order fractional differential equations of the type

$$
\begin{aligned}
& { }^{c} \mathcal{D}_{0+}^{q} u(t)+f(t, u(t))=0, q \in(n-1, n], n \geq 3, t \in(0,1), \\
& u^{\prime}(0)=\gamma u^{\prime}(\eta), u^{\prime \prime}(0)=0, u^{\prime \prime \prime}(0)=0, \ldots, u^{(n-1)}(0)=0, u(1)=\delta u(\eta),
\end{aligned}
$$

where $0<\eta, \gamma, \delta<1,{ }^{c} \mathcal{D}_{0+}^{q}$ is the Caputo fractional derivative. The function $f$ is assumed to be continuous and non-negative on $[0,1] \times[0, \infty)$ and may be nonlinear.

For convenience of the readers, we provide definitions of Riemann-Liouville fractional integral, fractional derivative, and some of their basic properties which will be helpful in the forth coming investigations.

Definition 1.1 ([13, 18]). For a function $\phi:(a, \infty) \rightarrow \mathbb{R}$, the RiemannLiouville fractional integral of order $\alpha>0$ is defined as

$$
\mathcal{I}_{a+}^{\alpha} \phi(t)=\frac{1}{\Gamma(\alpha)} \int_{a}^{t}(t-s)^{\alpha-1} \phi(s) d s,
$$

provided that the integral on right hand side exists.

For $\alpha, \beta \geq 0$, the fractional integral satisfies the semi group property

$$
\mathcal{I}_{0+}^{\alpha} \mathcal{I}_{0+}^{\beta} \phi(t)=\mathcal{I}_{0+}^{\alpha+\beta} \phi(t)=\mathcal{I}_{0+}^{\beta} \mathcal{I}_{0+}^{\alpha} \phi(t) \text { almost everywhere on }[0,1] .
$$

In addition, if $\phi \in C[0,1]$ or if $\alpha+\beta \geq 1$, then the identity is true for every $t \in[0,1]$.

Definition 1.2 ([13, 18]). The standard Riemann-Liouville fractional derivative of order $\alpha>0$ of a function $\phi:(a, \infty) \rightarrow \mathbb{R}$ is given by $\mathcal{D}_{a+}^{\alpha} \phi(t)=$ $\left(\frac{d}{d t}\right)^{n} \mathcal{I}_{a+}^{n-\alpha} \phi(t)$, where $a \in \mathbb{R}, n=[s]+1$, provided right hand side is pointwise defined on $(a, \infty)$. 
Definition 1.3. For a given function $\phi:(a, \infty) \rightarrow \mathbb{R}$, the Caputo fractional derivative of order $\alpha>0$ is defined by ${ }^{c} \mathcal{D}_{a+}^{\alpha} \phi(t)=\mathcal{I}_{a+}^{n-\alpha} \phi^{(n)}(t)$, where $a \in \mathbb{R}$, $n=[s]+1$.

Lemma $1.4([13])$. If $\alpha>\beta>0$, then ${ }^{c} \mathcal{D}_{0+}^{\beta} \mathcal{I}_{0+}^{\alpha} \phi(t)=\mathcal{I}_{0+}^{\alpha-\beta} \phi(t)$. In particular, if $m$ is positive integer and $\delta>m$, then $\frac{d^{m}}{d t^{m}}\left(\mathcal{I}_{0+}^{\delta} \phi(t)\right)=\mathcal{I}_{0+}^{\delta-m} \phi(t)$.

The following two lemmas play a fundamental role in obtaining an equivalent integral representation to the boundary value problem (1.1).

Lemma $1.5([13])$. Let $\alpha>0$. Then

$$
\begin{aligned}
\mathcal{I}_{0+}^{\alpha{ }^{c}} \mathcal{D}_{0+}^{\alpha} \phi(t) & =\phi(t)-\sum_{k=0}^{n-1} \frac{\phi^{k}(0)}{k !} t^{k} \\
& =\phi(t)+c_{1}+c_{2} t+c_{3} t^{2}+\cdots+c_{n} t^{n-1},
\end{aligned}
$$

where $c_{i}=\frac{\phi^{k-1}(0)}{(k-1) !}, i=1,2, \ldots, n$ and $n-1<\alpha \leq n$.

Lemma $1.6([13])$. For $\alpha>0$, the fractional differential equation ${ }^{c} \mathcal{D}_{0+}^{\alpha} \phi(t)=0$ has a general solution $\phi(t)=c_{1}+c_{2} t+c_{3} t^{2}+\cdots+c_{n} t^{n-1}$, where $c_{i} \in \mathbb{R}$, $i=1,2, \ldots, n$ and $n-1<\alpha \leq n$.

Now, applying $\mathcal{I}^{q}$ on both sides of the differential equation in (1.1), and using Lemma 1.5 and the boundary conditions in (1.1), the boundary value problem (1.1) can be equivalently written as an integral equation

$$
\begin{aligned}
u(t)= & -\mathcal{I}^{q} f(t, u(t))+\frac{\gamma}{1-\gamma} \mathcal{I}^{q-1} f(\eta, u(\eta))\left(\frac{1-\delta \eta}{1-\delta}-t\right) \\
& +\frac{1}{1-\delta}\left(\mathcal{I}^{q} f(1, u(1))-\delta \mathcal{I}^{q} f(\eta, u(\eta))\right) \\
= & \int_{0}^{1} k(t, s) f(s, u(s)) d s, t \in[0,1]
\end{aligned}
$$

where

$$
k(t, s)=\frac{1}{\Gamma(q)}\left\{\begin{array}{l}
-(t-s)^{q-1}+\frac{q \gamma}{1-\gamma}\left(\frac{1-\delta \eta}{1-\delta}-t\right)(\eta-s)^{q-2} \\
+\frac{1}{1-\delta}\left((1-s)^{q-1}-\delta(\eta-s)^{q-1}\right) \quad(s \leq t \leq 1, \eta \geq s), \\
\frac{q \gamma}{1-\gamma}\left(\frac{1-\delta \eta}{1-\delta}-t\right)(\eta-s)^{q-2} \\
+\frac{1}{1-\delta}\left((1-s)^{q-1}-\delta(\eta-s)^{q-1}\right) \quad(t \leq s \leq 1, \eta \geq s), \\
-(t-s)^{q-1}+\frac{1}{1-\delta}(1-s)^{q-1} \quad(s \leq t \leq 1, \eta \leq s), \\
\frac{1}{1-\delta}(1-s)^{q-1} \quad(t \leq s \leq 1, \eta \leq s) .
\end{array}\right.
$$

Clearly, we know $k(t, s) \geq 0$ on $(0,1) \times(0,1)$. 


\section{Upper and lower solutions method}

For the purpose of comparison results, consider the following nonlinear boundary value problem for fractional differential equation

$$
\begin{aligned}
& { }^{c} \mathcal{D}^{q} u(t)+h(t)=0, \quad t \in(0,1), n-1<q \leq n \\
& u^{\prime}(0)-\gamma u^{\prime}(\eta)=a_{1}, u^{\prime \prime}(0)=a_{2}, \ldots, u^{(n-1)}(0)=a_{n-1}, u(1)-\delta u(\eta)=b,
\end{aligned}
$$

where $b, a_{j} \in \mathbb{R}, j=1,2, \ldots, n-1$. The boundary value problem (2.1) is equivalent to the following integral equation

$$
u(t)=b \psi_{1}+a_{1} \psi_{2}+\sum_{j=3}^{n} \frac{a_{j-1}}{(j-1) !} \psi_{j}+\int_{0}^{1} k(t, s) h(s) d s, t \in[0,1],
$$

where $\psi_{1}=\frac{1}{1-\delta}, \psi_{2}(t)=-\frac{1}{1-\gamma}\left(\frac{1-\delta \eta}{1-\delta}-t\right), \psi_{j}(t)=-\left(\frac{1-\delta \eta^{j-1}}{1-\delta}-t^{j-1}\right)-$ $\frac{\gamma(j-1) \eta^{j-2}}{1-\gamma}\left(\frac{1-\delta \eta}{1-\delta}-t\right), j=3,4, \ldots, n$. Note that $\psi_{1}>0, \psi_{2}<0$ and $\psi_{j}(t) \leq 0$ on $(0,1)$ for $j=3,4, \ldots, n$. Hence, if $b \geq 0, a_{1} \leq 0, a_{j} \leq 0, j=2,4, \ldots, n$ and $h(t) \geq 0$ for $t \in[0,1]$, then any solution $u$ of the integral equation (2.2) satisfies $u(t) \geq 0$ on $(0,1)$. On the other hand, if $b \leq 0, a_{1} \geq 0, a_{j} \geq 0, j=2,4, \ldots, n$ and $h(t) \leq 0$ for $t \in[0,1]$, then any solution $u$ of the integral equation $(2.2)$ satisfies $u(t) \leq 0$ on $(0,1)$. Thus, we have the following comparison result.

Comparison results: (i) If ${ }^{c} \mathcal{D}^{q} u(t) \leq 0$ on $(0,1)$ for $q \in(n-1, n], n \geq 3$ such that $u^{\prime}(0) \leq \gamma u^{\prime}(\eta), u^{\prime \prime}(0) \leq 0, \ldots, u^{(n-1)}(0) \leq 0, u(1) \geq \delta u(\eta)$, then any solution $u$ of the linear problem $-{ }^{c} \mathcal{D}^{q} u(t)=h(t)$ satisfies $u \geq 0$ on $(0,1)$.

(ii) If ${ }^{c} \mathcal{D}^{q} u(t) \geq 0$ on $(0,1)$ for $q \in(n-1, n], n \geq 3$ such that $u^{\prime}(0) \geq$ $\gamma u^{\prime}(\eta), u^{\prime \prime}(0) \geq 0, \ldots, u^{(n-1)}(0) \geq 0, u(1) \leq \delta u(\eta)$, then any solution $u$ of the linear problem $-{ }^{c} \mathcal{D}^{q} u(t)=h(t)$ satisfies $u \leq 0$ on $(0,1)$.

In view of the comparison results, we introduce the definition of upper and lower solutions corresponding to the BVP (1.1) as follows:

Definition 2.1. A function $\alpha$ is called a lower solution of the BVP (1.1) if $\alpha \in C^{n-1}[0,1]$ and satisfies

$$
\begin{aligned}
& -{ }^{c} \mathcal{D}^{q} \alpha(t) \leq f(t, \alpha(t)), q \in(n-1, n], t \in(0,1) \\
& \alpha^{\prime}(0) \geq \gamma \alpha^{\prime}(\eta), \alpha^{\prime \prime}(0) \geq 0, \ldots, \alpha^{(n-1)}(0) \geq 0, \alpha(1) \leq \delta \alpha(\eta) .
\end{aligned}
$$

An upper solution $\beta \in C^{n-1}[0,1]$ of the $\operatorname{BVP}(1.1)$ is defined similarly by reversing the inequality.

Define $\bar{\beta}=\max \{\beta(t): t \in[0,1]\}$ and $\bar{\alpha}=\min \{\alpha(t): t \in[0,1]\}$.

Theorem 2.2. Assume that there exist lower and upper solutions $\alpha, \beta \in C[0,1]$ of the BVP (1.1) such that $\alpha \leq \beta$ on $[0,1]$. Assume that $f:[0,1] \times \mathbb{R} \rightarrow(0, \infty)$ is continuous and non-decreasing with respect to $u$ on $[0,1]$. Then the $B V P$ (1.1) has $C[0,1]$ positive solution $u$ such that $\alpha(t) \leq u(t) \leq \beta(t), t \in[0,1]$. 
Proof. Consider the modified BVP for fractional differential equations

$$
\begin{gathered}
-{ }^{c} \mathcal{D}^{q} u(t)=F(t, u(t)), q \in(n-1, n], n \geq 3, \quad t \in(0,1), \\
u^{\prime}(0)=\gamma u^{\prime}(\eta), u^{\prime \prime}(0)=0, \ldots, u^{(n-1)}(0)=0, u(1)=\delta u(\eta),
\end{gathered}
$$

where $[10,11]$

$$
F(t, u)= \begin{cases}f(t, \beta(t)), & \text { if } u \geq \beta(t) \\ f(t, u(t)), & \text { if } \alpha(t) \leq u \leq \beta(t) \\ f(t, \alpha(t)), & \text { if } u \leq \alpha(t)\end{cases}
$$

The BVP (2.3) is equivalent to the following integral equation

$$
u(t)=\int_{0}^{1} k(t, s) F(s, u(s)) d s, t \in[0,1],
$$

which can equivalently be written as an operator equation $(I-A) u(t)=0$, where $I$ is the identity operator and $A u(t)=\int_{0}^{1} k(t, s) F(s, u(s)) d s, t \in[0,1]$. By a solution of the BVP (2.3), we means a fixed point of the operator equation.

It should be noted that any solution $u$ of the BVP (2.3) such that $\alpha(t) \leq$ $u(t) \leq \beta(t), t \in[0,1]$, is a solution of the BVP (1.1). On the other hand, as an application of the Schauder's fixed point theorem, the BVP (2.3) has a solution if $F$ is continuous and bounded on $[0,1] \times R$. Further, for each fixed $t \in[0,1]$, the non-decreasing property of $f(t, u)$ with respect to $u$ implies that $F(t, u)$ is non-decreasing with respect to $u$ on $[\bar{\alpha}, \bar{\beta}]$ and

$$
f(t, \alpha(t)) \leq F(t, u) \leq f(t, \beta(t)),(t, u) \in[0,1] \times \mathbb{R} .
$$

We only need to show that $\alpha(t) \leq u \leq \beta(t), t \in[0,1]$, where $u$ is solution of the BVP (2.3). Define $m(t)=\alpha(t)-u(t), t \in[0,1]$, where $u$ is solution of the $\operatorname{BVP}(2.3)$, and in view of the boundary conditions, we obtain $m^{\prime}(0) \geq$ $\gamma m^{\prime}(\eta), m^{\prime \prime}(0) \geq 0, \ldots, m^{(n-1)}(0) \geq 0, m(1) \leq \delta m(\eta)$. Using the definition of lower solution and (2.5), we obtain

$$
\begin{aligned}
-{ }^{c} \mathcal{D}^{q} m(t) & =-\mathcal{D}^{q} \alpha(t)+\mathcal{D}^{q} u(t) \\
& \leq f(t, \alpha(t))-F(t, u(t)) \leq 0, q \in(n-1, n], t \in[0,1] .
\end{aligned}
$$

Hence, by comparison results $m(t) \leq 0, t \in[0,1]$. Similarly, we can show that $u(t) \leq \beta(t), t \in[0,1]$.

Now, we approximate the solution of BVP (1.1) via the generalized quasilinearization $[10,11]$. Choose a function $\phi(t, u)$ such that $\phi, \phi_{u}, \phi_{u u} \in C([0,1] \times$ $\mathbb{R}), \frac{\partial^{2}}{\partial u^{2}} \phi(t, u) \geq 0$ on $[\bar{\alpha}, \bar{\beta}]$ and

$$
\frac{\partial^{2}}{\partial u^{2}}[f(t, u)+\phi(t, u)] \geq 0 \text { on }[0,1] \times[\bar{\alpha}, \bar{\beta}] .
$$

Here we remark that the expression (2.6) is a weaker relation than convexity and such function $\phi$ always exists. For example, if $\max \left\{f_{u u}(t, u):(t, u) \in\right.$ $[0, T] \times[\bar{\alpha}, \bar{\beta}]\}=M$, then we may choose $\phi=\frac{M u^{2}}{2}$. 
Define $F^{*}:[0,1] \times \mathbb{R} \rightarrow \mathbb{R}$ by $F^{*}(t, u)=f(t, u)+\phi(t, u)$. Then, $F^{*} \in$ $C([0,1] \times \mathbb{R})$ and

$$
\frac{\partial^{2}}{\partial u^{2}} F^{*}(t, u) \geq 0 \text { on }[0,1] \times[\bar{\alpha}, \bar{\beta}] .
$$

The expression (2.7) implies that

$$
f(t, u) \geq f(t, y)+F_{u}^{*}(t, y)(u-y)-[\phi(t, u)-\phi(t, y)], t \in[0,1]
$$

for $u, y \in[\bar{\alpha}, \bar{\beta}]$. The non decreasing property of $\phi_{u}$ with respect to $u$ on $[\bar{\alpha}, \bar{\beta}]$, that is, $\frac{\partial^{2}}{\partial u^{2}} \phi(t, u) \geq 0$ yields

(2.9) $\phi(t, u)-\phi(t, y)=\phi_{u}(t, c)(u-y) \leq \phi_{u}(t, \bar{\beta})(u-y)$ for $u \geq y, t \in[0,1]$

where $u, y \in[\bar{\alpha}, \bar{\beta}]$ such that $y \leq c \leq u$. Substituting this into (2.8), we have

$$
f(t, u) \geq f(t, y)+\left[F_{u}^{*}(t, y)-\phi_{u}(t, \bar{\beta})\right](u-y) \geq f(t, y)+\lambda(u-y) \text { for } u \geq y,
$$

where $\lambda=\min \left\{0, \min \left\{F_{u}^{*}(t, \bar{\alpha})-\phi_{u}(t, \bar{\beta}): t \in[0,1]\right\}\right\}$. We note that $\lambda \leq$ $F_{u}^{*}(t, z)-\phi_{u}(t, \bar{\beta}) \leq f_{u}(t, \bar{\beta}): t \in[0,1]$.

Define $g:[0,1] \times \mathbb{R} \times \mathbb{R} \rightarrow \mathbb{R}$ by

$$
g(t, u, y)=f(t, y)+\lambda(u-y) .
$$

We note that $g(t, u, y)$ is continuous on $[0,1] \times \mathbb{R} \times \mathbb{R}$ and for $u, y \in[\bar{\alpha}, \bar{\beta}]$, using (2.10) and (2.11), we have

$$
\left\{\begin{array}{l}
f(t, u) \geq g(t, u, y) \text { for } u \geq y, \\
f(t, u)=g(t, u, u) .
\end{array}\right.
$$

Now, we develop the iterative scheme to approximate the solution.

Theorem 2.3. Under the hypothesis of Theorem 2.2, there exists a bounded monotone sequence of solutions of linear problems converging uniformly and quadratically to a solution of the problem (1.1).

Proof. As an initial approximation, we choose $w_{0}=\alpha$ and consider the linear problem

$$
\begin{aligned}
-{ }^{c} \mathcal{D}^{q} u(t) & =g\left(t, u(t), w_{0}(t)\right), q \in(n-1, n], t \in[0,1] \\
u^{\prime}(0) & =\gamma u^{\prime}(\eta), u^{\prime \prime}(0)=0, \ldots, u^{(n-1)}(0)=0, u(1)=\delta u(\eta) .
\end{aligned}
$$

In view of the definition of lower and upper solutions and (2.12), it follows that

$$
\begin{aligned}
& g\left(t, w_{0}(t), w_{0}(t)\right)=f\left(t, w_{0}(t)\right) \geq-{ }^{c} \mathcal{D}^{q} w_{0}(t), q \in(n-1, n], t \in[0,1], \\
& g\left(t, \beta(t), w_{0}(t)\right) \leq f(t, \beta(t)) \leq-{ }^{c} \mathcal{D}^{q} \beta(t), q \in(n-1, n], t \in[0,1],
\end{aligned}
$$

which imply that $w_{0}$ and $\beta$ are lower and upper solutions of (2.13). Hence, by Theorem 2.2, there exists a solution $w_{1} \in C[0,1]$ of $(2.13)$ such that $w_{0} \leq w_{1} \leq$ 
$\beta$ on $[0,1]$. Again, from (2.12) and the fact that $w_{1}$ is a solution of (2.13), we obtain

$$
-{ }^{c} \mathcal{D}^{q} w_{1}(t)=g\left(t, w_{1}(t), w_{0}(t)\right) \leq f\left(t, w_{1}(t)\right), q \in(n-1, n], t \in[0,1],
$$

which implies that $w_{1}$ is a lower solution of (1.1).

Similarly, we can show that $w_{1}$ and $\beta$ are lower and upper solutions of the linear problem

$$
\begin{aligned}
-{ }^{c} \mathcal{D}^{q} u(t)=g\left(t, u(t), w_{1}(t)\right), q \in(n-1, n], t \in[0,1] \\
u^{\prime}(0)=\gamma u^{\prime}(\eta), u^{\prime \prime}(0)=0, \ldots, u^{(n-1)}(0)=0, u(1)=\delta u(\eta) .
\end{aligned}
$$

Hence, by Theorem 2.2, there exists a solution $w_{2} \in C[0,1]$ of $(2.15)$ such that $w_{1} \leq w_{2} \leq \beta$ on $[0,1]$. Continuing in the above fashion, we obtain a bounded monotone sequence $\left\{w_{n}\right\}$ of solutions of linear problems satisfying

$$
w_{0} \leq w_{1} \leq w_{2} \leq w_{3} \leq \cdots \leq w_{n} \leq \beta \text { on }[0,1],
$$

where $w_{n}$ is a solution of the linear problem

$$
\begin{aligned}
-{ }^{c} \mathcal{D}^{q} u(t) & =g\left(t, u(t), w_{0}(t)\right), q \in(n-1, n], t \in[0,1] \\
& u^{\prime}(0)=\gamma u^{\prime}(\eta), u^{\prime \prime}(0)=0, \ldots, u^{(n-1)}(0)=0, u(1)=\delta u(\eta)
\end{aligned}
$$

and is given by

$$
w_{n}(t)=\int_{0}^{1} k(t, s) g\left(s, w_{n}(s), w_{n-1}(s)\right) d s, t \in[0,1] .
$$

The monotonicity and uniform boundedness of the sequence $\left\{w_{n}\right\}$ implies the existence of a pointwise limit $w$ on $[0,1]$ such that $w_{n} \rightarrow w$ uniformly. From the dominated convergence theorem, it follows that for any $t \in[0,1]$,

$$
\int_{0}^{1} k(t, s) g\left(s, w_{n}(s), w_{n-1}(s)\right) d s \rightarrow \int_{0}^{1} k(t, s) f(s, w(s)) d s .
$$

Passing to the limit as $n \rightarrow \infty,(2.17)$ yields $w(t)=\int_{0}^{1} k(t, s) f(s, w(s)) d s, t \in$ $[0,1]$, which implies that $w$ is a solution of (1.1).

To show that the convergence is quadratic, define $e_{n}(t)=w(t)-w_{n}(t), t \in$ $[0,1]$, where $w$ is a solution of (1.1). Then, $e_{n}(t) \geq 0$ on $[0,1], e_{n}(t) \rightarrow 0$ as $n \rightarrow \infty$ and from the boundary conditions, we have $e_{n}^{\prime}(0)=\gamma e_{n}^{\prime}(\eta), e_{n}^{\prime \prime}(0)=$ $0, \ldots, e_{n}^{(n-1)}(0)=0, e_{n}(1)=\delta e_{n}(\eta)$. Moreover, for every $t \in[0,1]$, we have

$(2.18)-{ }^{c} \mathcal{D}^{q} e_{n}(t)=F^{*}(t, w(t))-\phi(t, w(t))-f\left(t, w_{n-1}(t)\right)-\lambda\left(w_{n}(t)-w_{n-1}(t)\right)$.

Using the mean value theorem and the nondecreasing property of $\phi_{u}$, that is, $\phi_{u u} \geq 0$ on $[0,1] \times[\bar{\alpha}, \bar{\beta}]$, we obtain,

$$
\begin{aligned}
\phi(t, w(t)) & \geq \phi\left(t, w_{n-1}(t)\right)+\phi_{u}\left(t, w_{n-1}(t)\right)\left(w(t)-w_{n-1}(t)\right) \\
& \geq \phi\left(t, w_{n-1}(t)\right)+\phi_{u}(t, \bar{\alpha})\left(w(t)-w_{n-1}(t)\right), \\
F(t, w(t)) & =F\left(t, w_{n-1}(t)\right)+F_{u}\left(t, w_{n-1}(t)\right)\left(w(t)-w_{n-1}(t)\right)
\end{aligned}
$$




$$
\begin{aligned}
& +\frac{F_{u u}(t, \xi)}{2}\left(w(t)-w_{n-1}(t)\right)^{2} \\
\leq & F\left(t, w_{n-1}(t)\right)+F_{u}(t, \bar{\beta})\left(w(t)-w_{n-1}(t)\right) \\
& +\frac{F_{u u}(t, \xi)}{2}\left(w(t)-w_{n-1}(t)\right)^{2},
\end{aligned}
$$

where $w_{n-1} \leq \xi \leq w$. Hence,

$$
\begin{aligned}
& F(t, w(t))-\phi(t, w(t)) \\
\leq & f\left(t, w_{n-1}(t)\right)+\left[F_{u}(t, \bar{\beta})-\phi_{u}(t, \bar{\alpha})\right]\left(w(t)-w_{n-1}(t)\right) \\
& +\frac{F_{u u}(x, \xi)}{2}\left(w(t)-w_{n-1}(t)\right)^{2} .
\end{aligned}
$$

Hence, the equation (2.18) can be rewritten as

$$
\begin{aligned}
-{ }^{c} \mathcal{D}^{q} e_{n}(t) \leq & {\left[F_{u}(t, \bar{\beta})-\phi_{u}(t, \bar{\alpha})\right] e_{n-1}(t)+\frac{F_{u u}(t, \xi)}{2}\left(e_{n-1}(t)\right)^{2} } \\
& -\lambda\left(e_{n-1}(t)-e_{n}(t)\right) \\
\leq & {\left[F_{u}(t, \bar{\beta})-\phi_{u}(t, \bar{\alpha})-\lambda\right] e_{n-1}(t)+\lambda e_{n}(t)+\frac{F_{u u}(t, \xi)}{2}\left(e_{n-1}(t)\right)^{2} . }
\end{aligned}
$$

Since $\lambda \leq 0$ and $F_{u}(t, \bar{\beta})-\phi_{u}(t, \bar{\alpha})-\lambda \geq 0$, it follows that

$$
\begin{aligned}
-{ }^{c} \mathcal{D}^{q} e_{n}(t) & \leq\left[F_{u}(t, \bar{\beta})-\phi_{u}(t, \bar{\alpha})-\lambda\right]\left\|e_{n-1}\right\|+\frac{F_{u u}(t, \xi)}{2}\left\|e_{n-1}(t)\right\|^{2} \\
& \leq \rho\left\|e_{n-1}\right\|+d\left\|e_{n-1}\right\|^{2}, t \in[0,1],
\end{aligned}
$$

where $\rho=\max \left\{F_{u}(t, \bar{\beta})-\phi_{u}(t, \bar{\alpha})-\lambda: t \in[0,1]\right\} \geq 0$ and $d=\max \left\{\frac{F_{u u}(t, y)}{2}\right.$ : $y \in[\bar{\alpha}, \bar{\beta}]\}$. By comparison results, $e_{n}(t) \leq z(t), t \in[0,1]$, where $z(t)$ is a solution of the linear BVP

$$
\begin{aligned}
& -{ }^{c} \mathcal{D}_{0+}^{q} z(t)=\rho\left\|e_{n-1}\right\|+d\left\|e_{n-1}\right\|^{2}, q \in(n-1, n], n \geq 3 \\
& z^{\prime}(0)=\gamma z^{\prime}(\eta), z^{\prime \prime}(0)=0, \ldots, z^{(n-1)}(0)=0, z(1)=\delta z(\eta),
\end{aligned}
$$

which is given by

$$
e_{n}(t) \leq z(t)=\int_{0}^{1} k(t, s) d\left\|e_{n-1}\right\|^{2} \leq A\left\|e_{n-1}\right\|+B\left\|e_{n-1}\right\|^{2},
$$

where $A=\max \left\{\rho \int_{0}^{1} k(t, s) d s: t \in[0,1]\right\}$ and $B=\max \left\{d \int_{0}^{1} k(t, s) d s: t \in\right.$ $[0,1]\}$.

\section{References}

[1] S. Abbas and M. Benchohra, Upper and lower solutions method for impulsive partial hyperbolic differential equations with fractional order, Nonlinear Anal. Hybrid Syst. 4 (2010), no. 3, 406-413.

[2] B. Ahmad and J. J. Nieto, Existence results for a coupled system of nonlinear fractional differential equations with three-point boundary conditions, Comput. Math. Appl. 58 (2009), no. 9, 1838-1843. 
[3] Z. Bai, On positive solutions of a nonlocal fractional boundary value problem, Nonlinear Anal. 72 (2010), no. 2, 916-924.

[4] Z. Bai and H. Lu, Positive solutions for boundary value problem of nonlinear fractional differential equation, J. Math. Anal. Appl. 311 (2005), no. 2, 495-505.

[5] M. Benchohra and S. Hamani, The method of upper and lower solutions and impulsive fractional differential inclusions, Nonlinear Anal. Hybrid Syst. 3 (2009), no. 4, 433-440.

[6] M. Benchohra, S. Hamani, and S. K. Ntouyas, Boundary value problems for differential equations with fractional order and nonlocal conditions, Nonlinear Anal. 71 (2009), no. 7-8, 2391-2396.

[7] J. V. Devi, F. A. McRae, and Z. Drici, Generalized quasilinearization for fractional differential equations, Comput. Math. Appl. 59 (2010), no. 3, 1057-1062.

[8] J. V. Devi and Ch. Suseela, Quasilinearization for fractional differential equations, Commun. Appl. Anal. 12 (2008), no. 4, 407-417.

[9] C. S. Goodrich, Existence of a positive solution to a class of fractional differential equations, Appl. Math. Lett. 23 (2010), no. 9, 1050-1055.

[10] R. A. Khan, Multi-Point Boundary Value Problems for Fractional Differential Equations, Comm. Appl. Nonlinear Anal. 18 (2011), no. 3, 31-40.

[11] _ Existence and approximation of solutions to three-point boundary value problem for fractional differential equations, Electron. J. Qual. Theory Differ. Equ. 2011 (2011), no. 58,8 pp.

[12] R. A. Khan and M. Rehman, Existence of multiple positive solutions for a general system of fractional differential equations, Comm. Appl. Nonlinear Anal. 18 (2011), no. $1,25-35$.

[13] A. A. Kilbas, H. M. Srivastava, and J. Trujillo, Theory and Applications of Fractional Differential Equations, Elsevier Science, Amsterdam, 2006.

[14] V. Lakshmikantham and A. S. Vatsala, General uniqueness and monotone iterative technique for fractional differential equations, Appl. Math. Lett. 21 (2008), no. 8, 828834 .

[15] C. F. Li, X. N. Luo, and Y. Zhou, Existence of positive solutions of the boundary value problem for nonlinear fractional differential equations, Comput. Math. Appl. 59 (2010), no. $3,1363-1375$.

[16] S. Liang and J. Zhang, Positive solutions for boundary value problems of nonlinear fractional differential equation, Nonlinear Anal. 71 (2009), no. 11, 5545-5550.

[17] F. A. McRae, Monotone iterative technique and existence results for fractional differential equations, Nonlinear Anal. 71 (2009), no. 12, 6093-6096.

[18] I. Podlubny, Fractional Differential Equations, Academic Press San Diedo, 1999.

[19] M. Rehman and R. A. Khan, Positive solutions to nonlinear higher-order nonlocal boundary value problems for fractional differential equations, Abstract. Appl. Anal. 2010 (2010), 15 pp., doi:10.1155/2010/501230

[20] __ Existence and uniqueness of solutions for multi-point boundary value problems for fractional differential equations, Appl. Math. Lett. 23 (2010), no. 9, 1038-1044.

[21] M. Rehman, R. A. Khan, and N. Asif, Three point boundary value problems for nonlinear fractional differential equations, Acta Math. Sci. Ser. B Engl. Ed. 31 (2011), no. 4, 13371346.

[22] J. Sabatier, O. P. Agarwal, and J. A. Ttenreiro Machado, Advances in Fractional Calculus, Theoretical Developments and Applications in Physics and Engineering, Springer 2007.

[23] A. Shi and S. Zhang, Upper and lower solutions method and a fractional differential equation boundary value problem, Electron. J. Qual. Theory Differ. Equ. 2009 (2009), no. $30,13 \mathrm{pp}$. 
[24] J. Wang and H. Xiang, Upper and lower solutions method for a class of singular fractional boundary value problems with p-Laplacian operator, Abstr. Appl. Anal. 2010 (2010), Art. ID $97182412 \mathrm{pp}$.

[25] W. Zhong, Positive solutions for multipoint boundary value problem of fractional differential equations, Abstr. Appl. Anal. 2010 (2010), Art. ID 601492, 15 pp.

Department of Mathematics

UNIVERSITY OF MALAKAND

Chakadara Dir(L), Khyber Pakhtunkhwa, Pakistan

E-mail address: rahmatlipk@yahoo.com 